\title{
Miranda
}

Revue pluridisciplinaire du monde anglophone /

Multidisciplinary peer-reviewed journal on the English-

speaking world

$18 \mid 2019$

Guerre en poésie, poésie en guerre

\section{Mr. Smith Goes West : La portée politique du jeu de James Stewart dans le Western (1939-1964)}

\section{David Roche}

\section{OpenEdition}

Journals

Édition électronique

URL : http://journals.openedition.org/miranda/16500

DOI : 10.4000/miranda. 16500

ISSN : 2108-6559

Éditeur

Université Toulouse - Jean Jaurès

\section{Référence électronique}

David Roche, «Mr. Smith Goes West : La portée politique du jeu de James Stewart dans le Western (1939-1964) », Miranda [En ligne], 18| 2019, mis en ligne le 16 avril 2019, consulté le 16 février 2021 URL : http://journals.openedition.org/miranda/16500 ; DOI : https://doi.org/10.4000/miranda.16500

Ce document a été généré automatiquement le 16 février 2021.

\section{cc) (i) () $\Theta$}

Miranda is licensed under a Creative Commons Attribution-NonCommercial-NoDerivatives 4.0 International License. 


\title{
Mr. Smith Goes West : La portée politique du jeu de James Stewart dans le Western (1939-1964)
}

\author{
David Roche
}

1 Avec Gary Cooper, Henry Fonda et bien sûr John Wayne, James Stewart est certainement la star masculine qui a le plus marqué l'histoire du Western hollywoodien classique. Mais contrairement aux trois autres, sa présence dans le Western est déséquilibrée, concentrée quasi exclusivement sur la deuxième moitié de sa carrière Destry Rides Again / Femme ou démon (Universal, George Marshall, 1939) fait figure d'exception, tandis que of Human Hearts (MGM, Clarence Brown, 1938) n'est pas à proprement parler un Western mais plutôt un mélodrame historique aux airs de pastorale qui se déroule dans un territoire déjà américain (l'Ohio, devenu État en 1803) et qui se clôt pendant la guerre de Sécession. Visiblement, la présence de Stewart dans le Western n'avait rien d'une évidence pour ses contemporains, et ce n'est d'ailleurs qu'après le succès de Winchester '73 (Universal, Anthony Mann, 1950) que les producteurs de Broken Arrow / La Flèche brisée (20th Fox, Delmer Daves, 1950) ont décidé de sortir le film que Darryl Zanuck avait mis au placard (Davis 28). Ce tournant s'explique à la fois par la carrière de l'acteur et par le contexte culturel. En 1950, Stewart a 42 ans; il cherche aussi bien à s'adapter au vieillissement qu'à innover en tant qu'artiste. La crédibilité de Stewart en tant que héros de Western s'explique aussi par la redéfinition de la masculinité qui, selon Wendy Chapman Peek, a lieu aux ÉtatsUnis à la suite de la Seconde Guerre mondiale ; cette nouvelle masculinité ne serait plus tournée uniquement vers une démonstration de force mais se définirait par la capacité à mener à bien ses objectifs : il s'agit avant tout d'être "compétent " (Peek prend d'ailleurs Broken Arrow comme exemple) (Peek 206-19; voir aussi Mayer 155). L'image de Stewart, si elle était aux antipodes de la masculinité dure chère à Theodore Roosevelt incarnée par des acteurs comme Cooper et Wayne, est plus en accord avec cette masculinité dont le courage et la ténacité ne reposent pas entièrement sur le physique. 
2 Richard Dyer définit la «star image » comme un conglomérat d'éléments filmiques et paratextuels, comprenant les multiples rôles dans les films ainsi que les discours qui circulent dans les médias (articles, entretiens, publicités, etc.) $(60,63)$; la « star image » est quelque chose que l'industrie hollywoodienne tente de contrôler, mais elle dépasse au final l'image que veulent construire aussi bien la star que les studios. La «star image » de Stewart, c'est celle d'un homme du peuple, associé, selon son biographe Marc Eliot, aux « devoirs civiques " et à une "morale inébranlable " (31-32); le terme "folksy ", qui évoque l'homme du peuple rustique, revient souvent pour la décrire (Davis 31-32). Luc Moullet décrit Stewart comme "l'homme tel qu'il est » (123), capable d'interpréter aussi bien un personnage banal que d'exception (119), de se fondre dans un groupe (118). Stewart est, comme Henry Fonda, celui qui porte les valeurs populistes de la small town America, les valeurs de l'amabilité, de l'honnêteté et de la tradition associées à cette Amérique mythique resurgissant dans les années 1930. C'est cette image que l'on trouve, bien sûr, dans Mr. Smith Goes to Washington / Mr. Smith au sénat (Columbia, Frank Capra, 1939) mais aussi dans Destry Rides Again, dont les héros défendent les valeurs américaines - l'éducation et la justice - contre la corruption (qu'elle soit fédérale ou locale), avec bon sens, cœur et franchise. Selon Marguerite Chabrol, Stewart est aussi employé pour "dé-sophistiquer " certaines comédies screwball (216-17), adaptées de pièces de Broadway, telles que You Can't Take It With You / Vous ne l'emporterez pas avec vous (Columbia, Capra, 1938) et The Philadelphia Story / Indiscrétions (MGM, George Cukor, 1940). Selon l'historien du cinéma Robert Sklar, le screwball visait à faire oublier les problèmes des classes populaires dans les années 1930 en se moquant des riches mais aussi en les rendant ridicules et donc sympathiques (207). Stewart remplace ainsi le Britannique Laurence Olivier dans l'adaptation filmique de No Time for Comedy / Finie la comédie (Warner Bros., William Keighley, 1940), américanise la Hongrie d'Ernst Lubitsch dans The Shop Around the Corner / Rendez-vous (MGM, 1940) (Moullet 117) et doit, avec Destry Rides Again et The Philadelphia Story, "réhabiliter auprès du grand public américain» Marlene Dietrich et Katherine Hepburn en les rendant plus « humaine $[\mathrm{s}] »($ Coe 66).

Dans un article intitulé «The Star Auteur: Jimmy Stewart Out West», Alex Davis soutient que la Seconde Guerre mondiale a été un véritable tournant dans la vie et dans la carrière de la star. Celui-ci fait partie des acteurs américains comme Clark Gable et Henri Fonda qui partent au combat. L'industrie exploite alors cette décision pour renforcer l'image de la star comme homme du peuple et ambassadeur des valeurs américaines traditionnelles; elle essaye d'étouffer les traits qui ne collent pas à cette image, dont les plaintes de Stewart concernant les conditions de vie dans l'armée et le traumatisme qu'il subit suite à sa dernière mission de combat (Davis 31-34). De toute évidence, Hollywood s'emploie à maintenir l'image du Mr. Smith de 1939. Au retour de la guerre, Stewart affirme vouloir tourner dans des comédies et surtout pas dans des films de guerre; il s'illustre en tant que George Bailey dans un nouveau film de Capra, It's a Wonderful Life / La Vie est belle (RKO, 1946), retrouve aussi la small town America dans Magic Town (RKO, William A. Wellman, 1947) et revient à la screwball avec You Gotta Stay Happy / L'extravagante Mlle Dee (Universal, H.C. Potter, 1948). Mais en 1948, Stewart signe aussi sa première collaboration avec Alfred Hitchcock avec Rope / La Corde (Warner Bros.) ; elle sera suivie par trois productions Paramount, Rear Window / Fenêtre sur cour (1954), The Man Who Knew Too Much / L'homme qui en savait trop (1956) et enfin Vertigo / Sueurs Froides (1958). Hitchcock met progressivement à jour un versant plus sombre de Stewart, entraperçu, selon Marguerite Chabrol, dans le personnage plus 
fantaisiste de Macaulay Connor dans The Philadelphia Story (217): Rupert Cadell, instigateur malgré lui du meurtre commis par deux de ses étudiants; L.B. Jefferies, voyeur à ses heures perdues; et enfin Scottie Ferguson, épris d'une femme qui n'existe pas et dont il contribuera à la mort par deux fois. C'est ce versant plus sombre au potentiel violent qui sera aussi développé en parallèle chez Anthony Mann (Davis 28), de Winchester '73 avec son histoire de vengeance biblique opposant les frères McAdam, à The Naked Spur / L'appât (MGM, 1953) où Stewart incarne Howard Kemp, un chasseur de primes prêt à tout. Avec Mann, remarque Luc Moullet, «Stewart semble exiger que son personnage ait un passé trouble " (124); maintenant "c'est lui le violent, le dur, l'impitoyable. » (126) Les collaborations entre Stewart et John Ford du début des années 1960 - Two Rode Together / Les deux cavaliers (Columbia, 1961), The Man Who Shot Liberty Valance / L'homme qui tua Liberty Valance (Paramount, 1962) et Cheyenne Autumn / Les Cheyennes (Warner Bros., 1964) - mettront l'accent sur d'autres facettes: l'« inefficacité » de Mr. Smith ou «le cynisme et la vénalité » de l'homme de l'Ouest (Moullet 137 ; Davis 40-41).

4 Il ne s'agit bien entendu pas de proposer une vision téléologique de la carrière de Stewart du « folksy » vers la noirceur ${ }^{1}$, mais plutôt de constater, à la suite de Murray Pomerance (61-85), la coexistence dans l'image de Stewart de Mr. Smith et de son double plus sombre, ainsi que la manière dont certains traits passent en arrière-plan au profit d'autres, et sont sollicités, bien entendu, selon les rôles - en 1959, Stewart incarne ainsi un nouvel avatar de Mr. Smith, l'avocat modeste Paul Biegler en quête de justice dans Anatomy of a Murder / Anatomie d'un meurtre d'Otto Preminger. Ce qui m'intéresse plus particulièrement, c'est d'explorer comment s'exprime le potentiel politique de l'image «populiste » de Stewart à travers son jeu, dans quelques-uns de ses Westerns, notamment Destry Rides Again, Broken Arrow, The Naked Spur, The Man Who Shot Liberty Valance et Cheyenne Autumn. Car Stewart, ne l'oublions pas, est aussi celui qui se révolte au nom de ses valeurs. Comme Davis le souligne, «ce qui transparaît dans le personnage de Stewart [dans Bend of the River / Les Affameurs (Universal, Anthony Mann, 1952)] n'est pas le noble Destry, mais la détermination féroce de Mr. Smith au Sénat, cette fois-ci transformée en soif de vengeance $»^{2}$ (37, je traduis). À travers une analyse qui doit tout aux méthodologies élaborées par James Naremore, Jacqueline Nacache, Christian Viviani et d'autres, et qui puise d'ailleurs dans leurs analyses du jeu de Stewart, ainsi que dans celles de Luc Moullet, je vais tenter de répondre à la question suivante: comment le jeu de Stewart participe-t-il à interroger les injustices qui découlent de la construction de l'Ouest et de l'idéologie qui la sous-tend? Autrement dit, comment une approche qui s'inscrit dans la lignée de la politique des acteurs chère à Moullet peut-elle participer à une lecture culturelle et politique des films ?

5 Naremore décrit Stewart comme un acteur contemporain doué aussi bien pour un jeu tout en retenue que pour la pantomime, qui emploie des touches personnelles pour les moments émotionnels, par exemple quand Stewart mord son poing pour signifier le désarroi de son personnage (286). Viviani cite Stewart en exemple d'un comédien qui, comme Robert Mitchum, Robert De Niro ou Al Pacino, «développe un catalogue de gestes expressifs, relevant le plus souvent du registre du théâtral, qu'il utilise de manière delsartienne sans pour autant être totalement un pur adepte de Delsarte " (140). Comme François Delsarte, Stewart vise à exprimer le naturel par un geste précis ou une inflexion de voix singulière, loin de cette codification des gestes qu'est le delsartisme promu par l'acteur américain Steele MacKay et ses disciples (Damour 19, 30). Au sujet du jeu de Stewart dans Rear Window, Naremore écrit : «Son interprétation 
est certainement de celles le plus souvent liées au médium cinématographique : concentrée sur le visage et la partie supérieure du corps, elle met l'accent sur les tonalités de la peau, l'expression des yeux et le grain de la voix.» (284) Naremore insiste également sur l'importance de la silhouette grande et maigre («lankiness ») de Stewart qui évoque la vertu populiste d'un Lincoln, et affirme que les films de la seconde période de sa carrière comme The Man Who Shot Liberty Valance ont énormément joué sur son apparence filiforme et fragile pour augmenter la tension dramaturgique d'une scène (Naremore 286, 289). Compte tenu du travail précis que Moullet a proposé sur la gestuelle de Stewart, l'analyse qui suit va se concentrer sur les trois éléments soulignés par Naremore : posture, voix et regard.

\section{Posture et mouvement}

Le travail sur les postures et mouvements de Stewart dans Destry Rides Again est, dans l'ensemble, assez minimal. Sa grande taille (chez Frenchie, il tient à peine dans le cadre de la porte) fait qu'il domine les autres personnages, renforçant son statut de repère moral pour les spectateurs ; ainsi, il est toujours visible, même pris dans le tumulte de la foule dans le saloon et filmé en plongée. Ses postures - mains sur les hanches ou accoudé contre un poteau - évoquent cette douceur presque «féminine » associée aux valeurs pacifistes ${ }^{3}$ : il est celui qui descend de la calèche avec un canari et un parasol plutôt qu'avec des armes. Ses gestes plus violents - quand il tape un des hommes de Kent sur le dos et surtout quand il tire avec les revolvers de Creepy - sont effectués avec une nonchalance qui accompagne son ton ironique. L'association du corps de Stewart à la féminité est exploitée dès sa scène d'introduction, où il perd l'équilibre dans une calèche et tombe sur Janice Tyndall. Elle l'est plus longuement lors de son affrontement avec le personnage incarné par Marlene Dietrich, qui donne lieu à un combat sur le mode du slapstick; la scène met en avant ses longues jambes et longs bras qui lui permettent de maintenir Dietrich à distance, mais qui donnent tout autant l'impression d'une perte d'équilibre inéluctable, voire d'une fragmentation du corps en plusieurs morceaux tant les membres paraissent se dissocier (Fig. 1). Le corps de Stewart semble ainsi incarner à la fois la droiture morale (et participe ainsi à sa fonction de baromètre moral) et la difficulté de la maintenir (et donc la limite du projet de Destry).

Fig. 1 


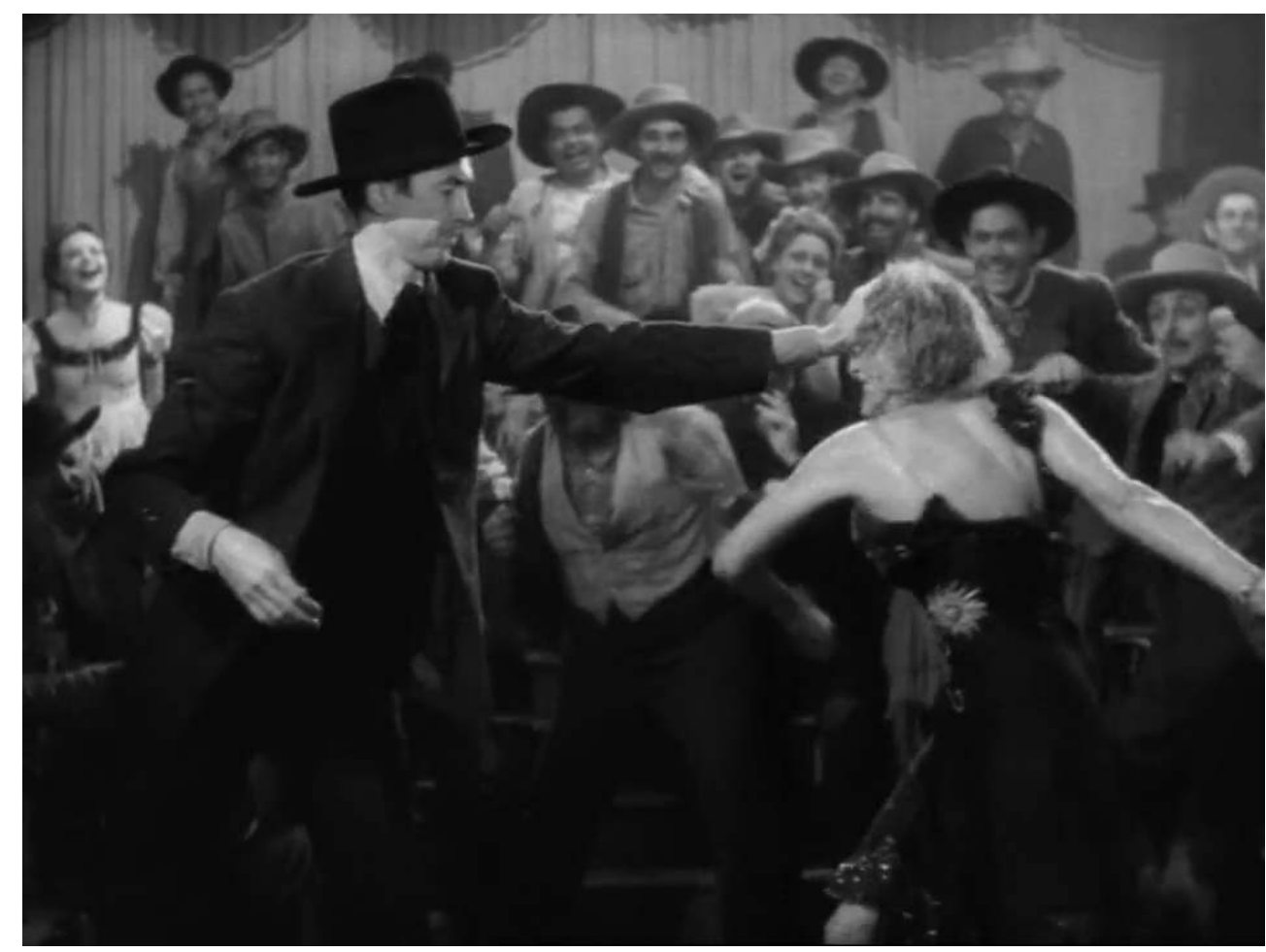

Destry Rides Again (George Marshall, 1939) : Destry tente tant bien que mal de contenir Frenchy.

7 Dans Broken Arrow, la haute taille de Stewart (1m 90) signifie également la droiture de Jeffords, mais il la partage avec Jeff Chandler (1m 93) qui interprète Cochise. Sa démarche et ses mouvements sont généralement calmes, évoquant à la fois la confiance en soi de l'homme de l'Ouest mais aussi la confiance qu'il souhaite inspirer en autrui ; elle participe pleinement de la caractérisation de Jeffords comme celui qui souhaite apporter la paix. Stewart emploie ses mains à plusieurs reprises, de manière à améliorer la communication avec les Apaches, mais aussi pour signifier son agacement envers les villageois, et enfin pour se battre. La gestuelle ne laisse donc aucune place à l'ambiguïté concernant la fonction de la star : Stewart est bien le repère moral autour duquel les autres personnages s'organisent de façon assez binaire.

8 Dans The Naked Spur, Stewart fait appel à son passé athlétique (il était sportif à Princeton) en adoptant dès le départ la posture, la démarche et la gestuelle de l'homme de l'Ouest; la première scène présente Howard Kemp comme un - professionnel capable de surprendre Tate et lui confisquer son fusil. Agile, débrouillard, efficace, maîtrisé, ses qualités sont évoquées dans le titre puisque c'est en jetant un éperon au visage de Ben que Kemp sauvera Lina. Ses mouvements sont brutaux et explosifs, comme quand il pousse son prisonnier, Ben, qui tente de monter les hommes les uns contre les autres, ou quand il sauve Lina d'un guerrier Blackfoot qu'il rosse à coups de rocher. Si Kemp maîtrise le plus souvent ses adversaires (lors de sa bagarre avec Roy ou quand Ben tente de s'échapper), le film joue néanmoins sur la possibilité du déséquilibre du corps de Stewart afin d'exprimer les faiblesses du personnage. Dès le départ, Kemp ne parvient pas à grimper sur le rocher (Fig. 2) et doit donc accepter l'assistance de Roy; plus tard, il chute de cheval suite à une fourberie de Ben, ce qui l'amènera à révéler sa vulnérabilité à la fois physique (il boite) et psychologique (en proie au délire, il fait un cauchemar dans lequel figure sa bien-aimée perdue). Le fait qu'il parvienne à grimper sur le rocher pour sauver Lina à la fin du film signale ainsi sa 
rédemption: son physique est mis au service non plus de sa cupidité (ambition maintenant prise en charge par Roy qui avait initialement exprimé son désir pour Lina), mais de valeurs plus nobles comme la chevalerie et l'amour.

\section{Fig. 2}

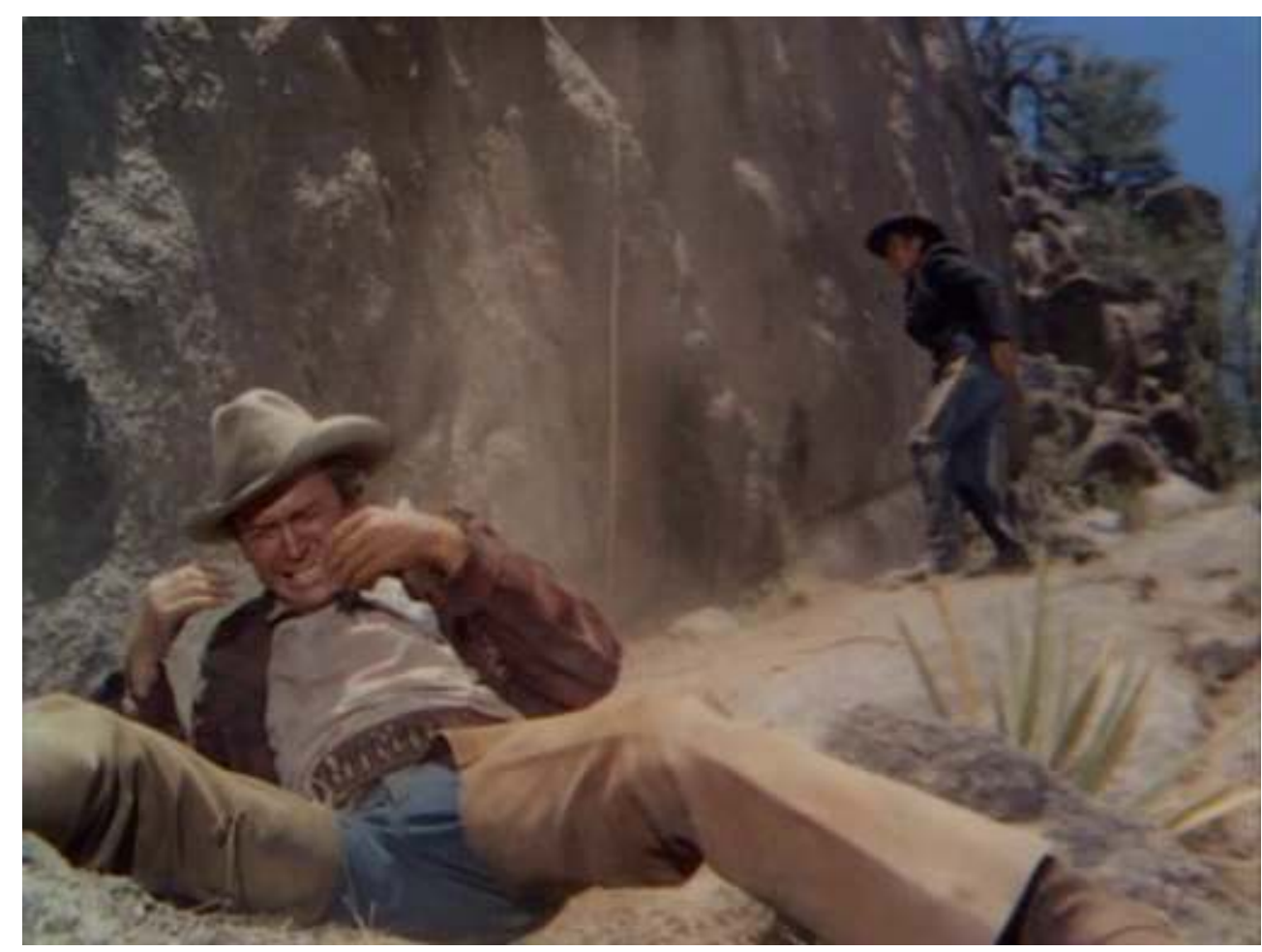

The Naked Spur (Anthony Mann, 1953) : Kemp chute après avoir tenté de grimper une falaise.

9 The Man Who Shot Liberty Valance fait grand usage de la posture de Stewart. Si le Sénateur est présenté comme un homme grand et droit à la démarche lente mais confiante, bref, à la hauteur de l'institution qu'il représente, le jeune Stoddard est celui qui ne cesse d'être mis au sol par les hommes de l'Ouest qu'il rencontre: Doniphon, mais surtout Valance qui le fouette, lui fait un croche-pied et l'oblige à s'accroupir pour ramasser son revolver (Fig. 3). La relation entre ces trois corps est établie lors de l'altercation entre Doniphon et Valance dans le restaurant où le morceau de viande au sol devient une métonymie du corps de Stewart; plus tard, le revolver des hommes de l'Ouest amène Stoddard à adopter des postures inconfortables. Stewart est, bien plus encore que dans The Naked Spur, ce corps qui menace de s'écrouler. Lors de leur première rencontre, Doniphon tient Stoddard par le col d'abord pour le maîtriser, ensuite pour l'empêcher de s'évanouir. La capacité de Stoddard de mettre au sol Doniphon est alors le signe qu'il contient lui aussi une part de l'homme de l'Ouest - ce sera d'ailleurs le deuxième argument que Doniphon convoque pour soutenir la candidature de l'avocat. D'une "délicatesse "si féminine" ", Stoddard est, selon Aimé Agnel, parvenu à «s'approprier les vertus » de l'homme de l'Ouest tout en conservant un « courage éthique [...] qui trouve son fondement dans la part la plus féminine et la plus obscure de son être » (159-60). Le travail sur la droiture de Stewart est accentué par l'emploi très genré des costumes: le costume noir du Sénateur assoit une dignité que le jeune Stoddard perd quand il porte le tablier, dont la souplesse renforce l'impression de déséquilibre ; le jeune Stoddard acquiert une certaine dignité quand il 
endosse un costume gris dans la salle de classe ou lors des élections. Le déséquilibre qui menace le corps de Stewart, c'est donc l'état sauvage qui menace le processus de civilisation. Replacé dans l'ordre chronologique, la transformation du corps de Stewart, en réussissant à synthétiser Est et Ouest, vient alors signifier la construction de la nation dont les trois personnages masculins incarnaient chacun une étape (Agnel 151) : d'une blancheur hystérique (Stewart donne au jeune Stoddard des gestes beaucoup plus nerveux qui rappellent certains de ses personnages chez Mann) qui se perd dans les intérieurs clairs, il devient une présence noire, sereine et solide qui interpelle le regard, en particulier à travers la posture lincolnienne qui clôt le film (Fig. 4) et qui n'est pas sans rappeler celle de Henri Fonda quand il est assis à son bureau ou dans une chaise lors du procès dans Young Mister Lincoln.

Fig. 3

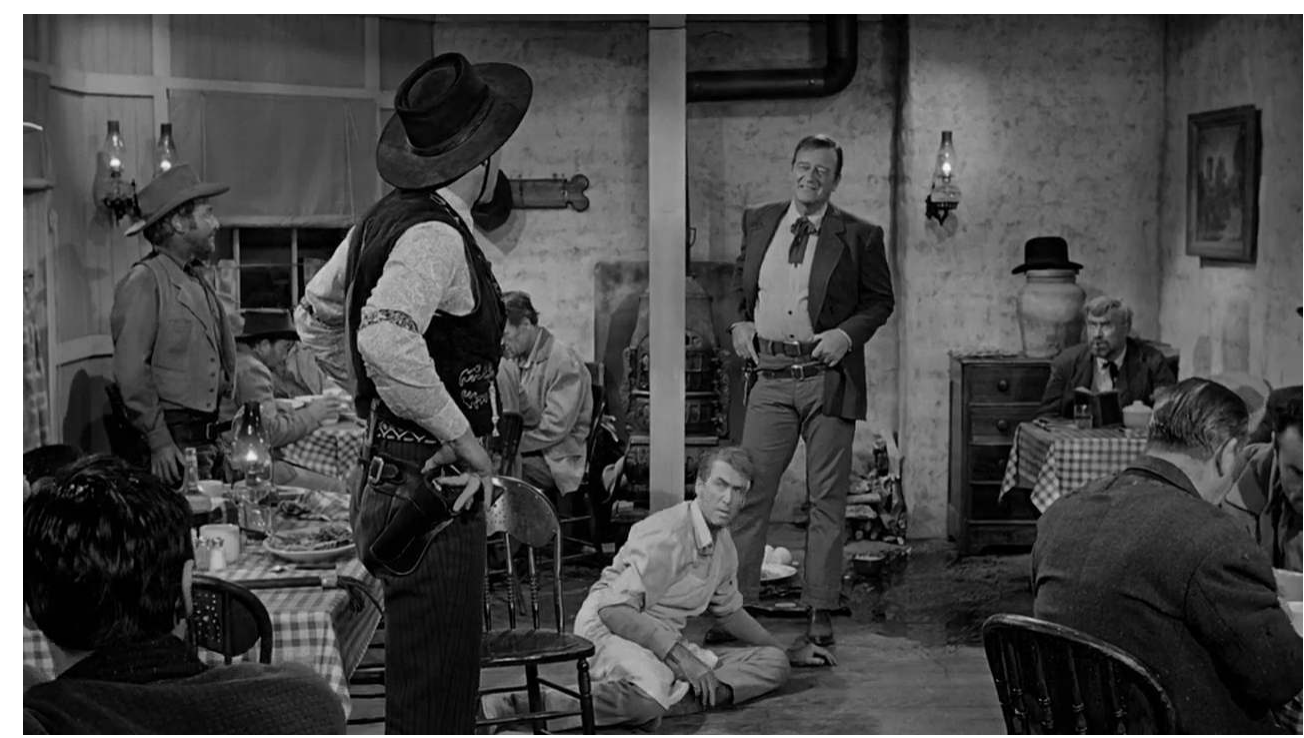

THE MAN WHO SHOT LIBERTY VALANCE (JOHN FORD, 1962) : STODDARD, AU SOL, ENTRE VALANCE ET DONIPHON. 
Fig. 4

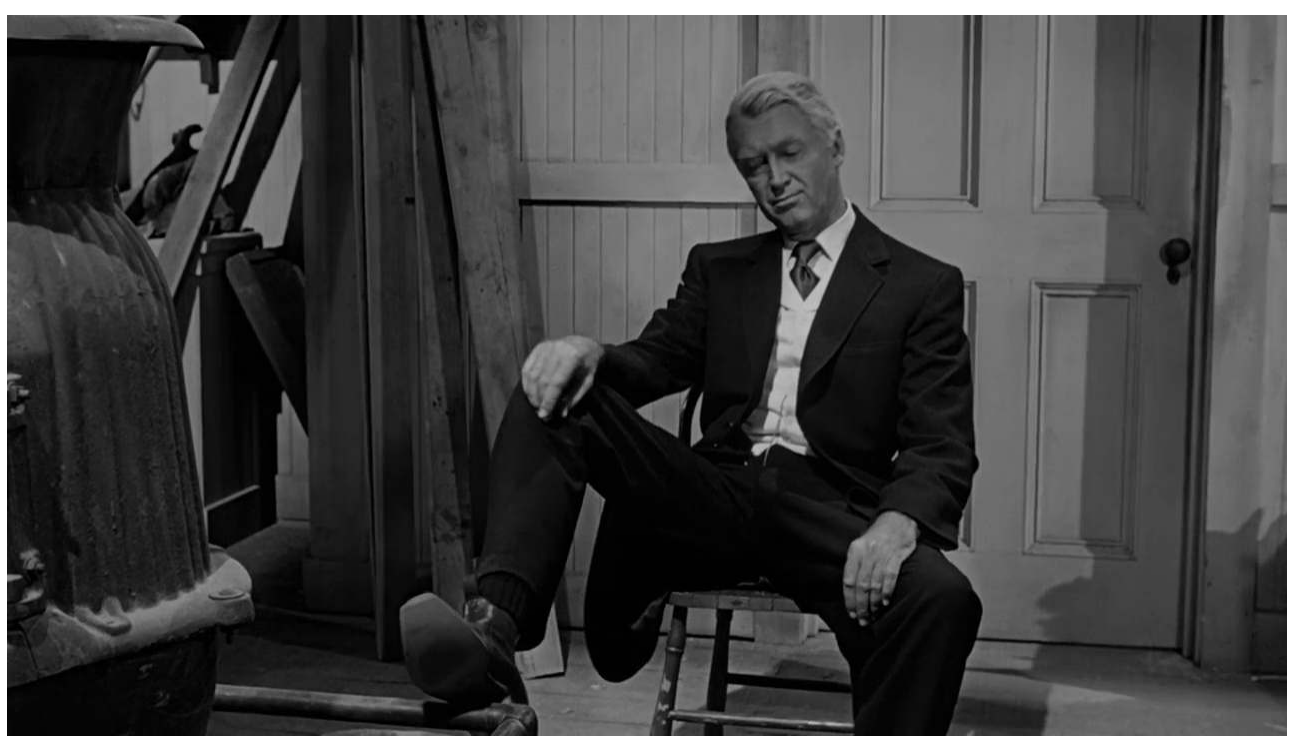

THE MAN WHO SHOT LIBERTY VALANCE (JOHN FORD, 1962) : LE SÉNATEUR STODDARD DANS UNE POSTURE LINCOLNIENNE.

Dans Cheyenne Autumn, la posture recroquevillée et la gestuelle plus lente de Stewart évoquent en premier lieu le vieillissement, mais le film reprend aussi le jeu ironique de Destry Rides Again : la posture de Stewart indique la nonchalance d'Earp, qui tire sur un Texan en restant assis à table; il assomme ledit Texan, puis lui tapote la jambe qu'il vient de soigner; et ses longues jambes, initialement dissimulées sous la table ou cachées derrière le bar, prennent bien trop de place dans la calèche qu'il occupe avec Doc Holliday, le déséquilibre figurant le chaos de la « drôle d'armée » (" motley army ») et annonçant le basculement de la calèche à venir. Le cabotinage de Stewart participe largement à la déconstruction de la figure iconique de l'Ouest et du Western (Moullet 137).

\section{Voix}

11 Le grain de la voix de Stewart participe largement du caractère « folksy » de son image. Doucereuse, légèrement nasillarde, moins aiguë et plus profonde que celle de Henry Fonda, elle invoque déjà un sujet proprement américain, entre enfance et âge adulte. Le caractère « folksy » est renforcé par une prononciation parfois à la limite du « Southern drawl » (qui a tendance à diviser un son de voyelle en deux), alors même que Stewart est originaire de la Pennsylvanie et a fait ses études à Princeton. Son élocution est faite de modulations larges et fluides, mais il est aussi capable de balbutier d'une voix chevrotante comme dans You Can't Take It With You / Vous ne l'emporterez pas avec vous (Columbia, Capra, 1938) (Moullet 113-14) ou de délivrer un phrasé plus « clipped» (sec) et sophistiqué (le cachet Princeton, sans doute) qui tend vers celui d'un Cary Grant et que Stewart déploie en particulier dans le registre comique (esprit, ironie, etc.). C'est cette amplitude qui permet à la voix de Stewart de passer de la tendresse et la compassion à une révolte tout aussi sincère et passionnée, en passant par un humour bienveillant ou une ironie laconique. 
12 Dans Destry Rides Again, Stewart donne au héros une voix douce qui respire l'intelligence et inspire la confiance, tout en employant un dialecte rural typique des représentations de l'Ouest ("Howdy », "them first impressions»). Comme Lincoln, et notamment celui de son ami Henry Fonda apparu sur les écrans quelques mois plus tôt dans Young Mister Lincoln / Vers sa destinée (20th Fox, John Ford, 1939), Destry se plaît à raconter des histoires de personnes qu'il a/aurait connues en guise de leçons de vie, sur un ton souvent chantant et enjoué. En conversation, la voix de Destry/Stewart s'adapte à son interlocuteur : ton doux quand il s'adresse au fils Claggett qui, s'il est initialement attiré par les armes, sera conquis par le pacifisme de Destry et canalisera sa colère dans la confection de porte-serviettes en bois; ton plus romantique avec Frenchy, interprétée par Marlene Dietrich, qui joue une saloon girl forcément au cœur d'or ; ton menaçant envers Creepy, véritable danger public qui tire son revolver dans la rue; et ton plus méfiant avec Kent, interprété par Brian Donlevy (l'ignoble Barshee responsable de la mort de la mère de Jesse James dans le film éponyme de Henry King sorti plus tôt dans l'année), à nouveau dans la peau d'un malfrat aux allures de gangster. La voix de Stewart nous offre donc un repère clair qui renforce notre tendance à nous identifier à la star et fonctionne comme une sorte de baromètre moral permettant de jauger la valeur de chaque personnage. Plus généralement, elle remet en question les valeurs de l'Ouest fondées sur la violence, comme lors de cette conversation avec le sheriff Dimsdale où Stewart emploie un ton d'abord doux puis ensuite plus ferme: « My pa had these on in Tombstone. He got shot in the back. [...] Law and order. [...] My pa did it the old way, and I'm going to do it a new way. » Néanmoins, Destry Rides Again montre les limites de l'éloquence de Mr. Smith dans le Far Ouest - et de l'ironie déployée initialement par Destry, souvent avec succès, pour désamorcer la violence - et anticipe à ce titre The Man Who Shot Liberty Valance. Quand son ami le Sheriff Washington Dimsdale est mourant, Destry est incapable de terminer son histoire et la voix de Stewart se fait chevrotante. Destry devra alors endosser les valeurs de l'Ouest malgré lui pour libérer la ville de la corruption, et pour ce faire, Stewart devra en passer par l'aboiement et le silence, registres plus en adéquation avec la figure de l'homme de l'Ouest.

13 La voix de Stewart occupe une place centrale dans Broken Arrow à travers la voix off. Jeffords le narrateur exprime sur un ton doux et presque mélancolique (que la fin du récit justifiera) sa lassitude de la violence de l'Ouest et son expérience de l'empathie pour les victimes apaches. Ceci est particulièrement sensible lors de sa rencontre avec le jeune Apache quand Jeffords avoue que l'idée qu'une mère apache pouvait ressentir de la peine à la perte d'un enfant ne lui était jamais venue à l'esprit, mais aussi quand il exprime son horreur devant la punition infligée par les guerriers apaches au scalpeur, sa peur quand il se rend chez les Apaches pour la première fois, ou encore sa douleur suite à la mort de Sonseeahray. Stewart déploie un registre beaucoup plus large pour le Jeffords du temps du récit. La frustration et la colère de l'homme de l'Ouest s'expriment à plusieurs reprises dans le premier acte du film (notamment quand il s'oppose aux opinions des villageois blancs), Stewart augmentant le volume, montant dans les aigus et parfois accroissant le rythme d'élocution. C'est tout particulièrement le cas quand il donne une leçon d'histoire à Ben slade qui lui reproche d'être trop proche des Apaches :

Hold on, let's get the facts straight. Cochise didn't start this war. A snooty little Lieutenant fresh out of the East started it. He flew a flag of truce which Cochise 
honored and then he hanged Cochise's brother and five others under the flag. [...] You wanna know why I didn't kill that Apache boy? Well for the same reason I wouldn't kill your boy or scout for the army. I'm sick and tired of all this killing.

15 C'est bien la voix de Mr. Smith qui s'érige en défenseur des valeurs de bon sens que nous retrouvons dans Broken Arrow. Plus tard, la voix de Stewart se fait calme et ferme quand il présente la proposition de Cochise aux villageois, agressive quand il défend l'honneur de Cochise dans le salon. Le changement indique alors le basculement de l'homme de l'Ouest touché au vif qui agit selon un sens moral personnel et naturel vers l'homme politique qui vise à inspirer confiance.

Dans les films de Mann, l'amplitude vocale de Stewart va permettre d'évoquer des ambiguïtés psychiques et morales, si bien que la cible de la critique véhiculée par Stewart est finalement le caractère pathologique de l'homme de l'Ouest (d'une certaine manière, c'est aussi ce que John Ford fait avec Fonda dans Fort Apache [1948] et avec Wayne dans The Searchers [1956]). La voix du justicier se retourne contre elle-même. Dans The Naked Spur, Stewart pose une voix de l'homme de l'Ouest archétypique : avec un ton plus grave et un phrasé plus monocorde, cette voix, habitée de plus de silences qu'à son habitude, prononce ordres et questions. C'est sur un ton calme que Kemp ordonne à Roy de les quitter, et sa voix demeure calme quand le groupe se retrouve face à face aux Blackfeet. Les ruptures dans la tonalité quand Stewart hurle ne signifient pas, ici, la révolte contre l'injustice mais, au contraire, son indifférence: "Quit actin' like we was friends! It's him they're paying the reward for! », si bien que Ben, le prisonnier encore plus franchement hystérique que Kemp, parait dans un premier temps plus dans l'empathie que Kemp (ce qui explique qu'il ait pu séduire Lina). Face à un Ben qui le psychanalyse ("Choosing the way to die, what's the difference? Choosing the way to live, that's the part. That's what's eating you, ain't it, Howie? »), Kemp, en colère, exige le silence avec deux mots (« Shut up! »). Ces ruptures annoncent bien entendu le hurlement de Kemp quand il délire à cause de la fièvre, Stewart faisant alors dérailler sa voix dans les aigus, puis son hurlement hystérique quand il ordonne à Ben de fuir en haletant. Ces fissures dans la voix de l'homme de l'Ouest expriment son trauma, bien sûr, mais c'est aussi à travers elles que Mr Smith va finir par se glisser, Stewart faisant alors appel à son célèbre registre «folksy » quand il parle avec Lina sur un ton nostalgique de sa vie pastorale d'antan. Le travail sur la voix de Stewart décrit alors un cheminement parallèle à celui, spatial, des personnages : l'homme de l'Ouest à la masculinité dure est un masque, un mécanisme de défense, qui signifie le refoulement du Mr. Smith populiste à travers des symptômes bien connus (anxiété, halètement, etc.).

Chez Ford, le jeu de Stewart sert aussi principalement une critique de son propre personnage, mais Liberty Valance et Cheyenne Autumn emploient deux approches différentes. Le film de 1962 invoque Mr. Smith et le récent Anatomy of a Murder pour nous positionner du côté de l'avocat dans la lutte pour la justice. Comme dans Broken Arrow, le film contraste la voix plus posée, solennelle mais aussi plus rauque du personnage plus âgé qui narre l'histoire - le Sénateur conserve néanmoins le caractère "folsky " grâce à un accent rappelant le "Southern drawl ", signifiant sa proximité avec ses origines que démontre son retour pour la mort de Tom Doniphon - à celle plus instable du personnage plus jeune. Le la est donné dès le premier affrontement entre Stoddard et Valance, lors duquel l'avocat hurle : «I'm an attorney in law. I'll see you in jail for this. »; l'explosivité de l'homme de l'Est contraste avec le sang froid de l'homme 
de l'Ouest. Il en est de même quand Stoddard rencontre Doniphon et bredouille cette fois-ci d'une voix douce et chevrotante : «I've got something to do. [...] I don't want a gun. I don't wanna kill him. I want to put him in jail. » Stoddard hurle à nouveau lors de la scène du steak: « Nobody fights my battles! ». Sur la Frontière, la voix de la raison est, paradoxalement, la voix de l'hystérique, et contrairement à chez Mann, il s'agit ici de l'hystérie de l'homme de l'Est «féminisé » (Doniphon le décrit comme un «lady's man »); il est significatif que Stoddard s'en prenne aussi à Hallie et ensuite à Doniphon quand il est question d'amour, ou quand il s'agit de cautionner le mensonge qui le transforme en homme de l'Ouest (Doniphon répond d'ailleurs : «You talk too much. »). La voix de Stewart se stabilise quand Stoddard a l'occasion de faire corps avec l'institution, qu'il lise un texte de loi, explique le processus d'amendement de la Constitution ou le déroulement des élections; son ton reprend confiance et s'élève quand il explique ce que signifie l'intégration à la nation: "Statehood means the protection of our farms and our fences, and it means schools for our children, and it means progress for the future. » Les modulations de voix de Stewart décrivent ainsi les tensions entre civilisation et sauvagerie, Est et Ouest, féminin et masculin, qui, dans le Western classique, sont souvent réparties entre personnage masculin et féminin (les Yorke dans Rio Grande [John Ford, 1950] pour citer un exemple paradigmatique) (Slotkin 182, 289, 359-61).

Dans Cheyenne Autumn, Stewart propose, au contraire, un phrasé assez monocorde, désabusé ; dans un premier temps, il marmonne, le cigare à la bouche, ses propos ponctués juste d'une menace agacée envers un Texan, et plus tard, lors de la présentation de son "plan de campagne » qui consiste à éviter les Apaches, quand le vacarme environnant l'oblige à hurler. L'échec de Stoddard s'inscrit, en quelque sorte, dans ce qui est finalement une inversion de Mr. Smith : l'avocat est le défenseur d'un système fédéral plutôt que des valeurs de bon sens de l'Amérique profonde; ce sont au contraire ces valeurs-là qu'il va devoir apprendre. Le Wyatt Earp de Cheyenne Autumn sert, quant à lui, à une démythification d'une icône de l'Ouest au niveau moral (manque de courage, d'empathie et de sens de l'honneur) plus que physique (après tout, Earp demeure un tireur habile).

\section{Regard}

19 Hitchcock l'avait compris, Stewart est aussi un regard : perçant, malicieux, inquiet (le regard vers la gauche noté par Moullet [119]). Si le casting de Stewart dans le rôle du photographe voyeur de Rear Window et les très gros plans du regard du détective dans Vertigo l'ont mis en évidence, le regard de Stewart joue un rôle fondamental dans les implications politiques de son jeu parce qu'il peut évoquer l'émerveillement face aux valeurs américaines, la générosité envers son prochain ou se faire accusateur contre les forces de la corruption.

20 Dans Destry Rides Again, le regard de Stewart accompagne la voix dans sa fonction de baromètre moral face à autrui. Quand Destry raconte ses histoires, le regard de Stewart, souvent dirigé vers le sol, évite ses interlocuteurs, du moins jusqu'à ce qu'il lève les yeux pour jauger l'effet de son histoire, autrement dit pour déterminer si son public a compris la morale. Le côté fantaisiste et déconnecté de Destry n'est donc qu'un leurre son regard fuyant est sincère uniquement quand il traduit l'embarras de se trouver en situation intime avec Frenchy. Car le regard perçant de Stewart, souvent mis en avant 
par le chapeau de cowboy, est déjà celui de l'enquêteur, celui qui cherche à démêler la corruption et à résoudre le meurtre du Sheriff précédent : Destry regarde ses suspects droit dans les yeux quand il les questionne; c'est le regard de Stewart qui traduit sa prise de conscience de la culpabilité du maire (Fig. 5). Rien d'étonnant, donc, à ce que l'échec de Destry soit aussi signifié par le regard: le chapeau de Stewart cache son visage quand il est contraint à reprendre les armes.

Fig. 5

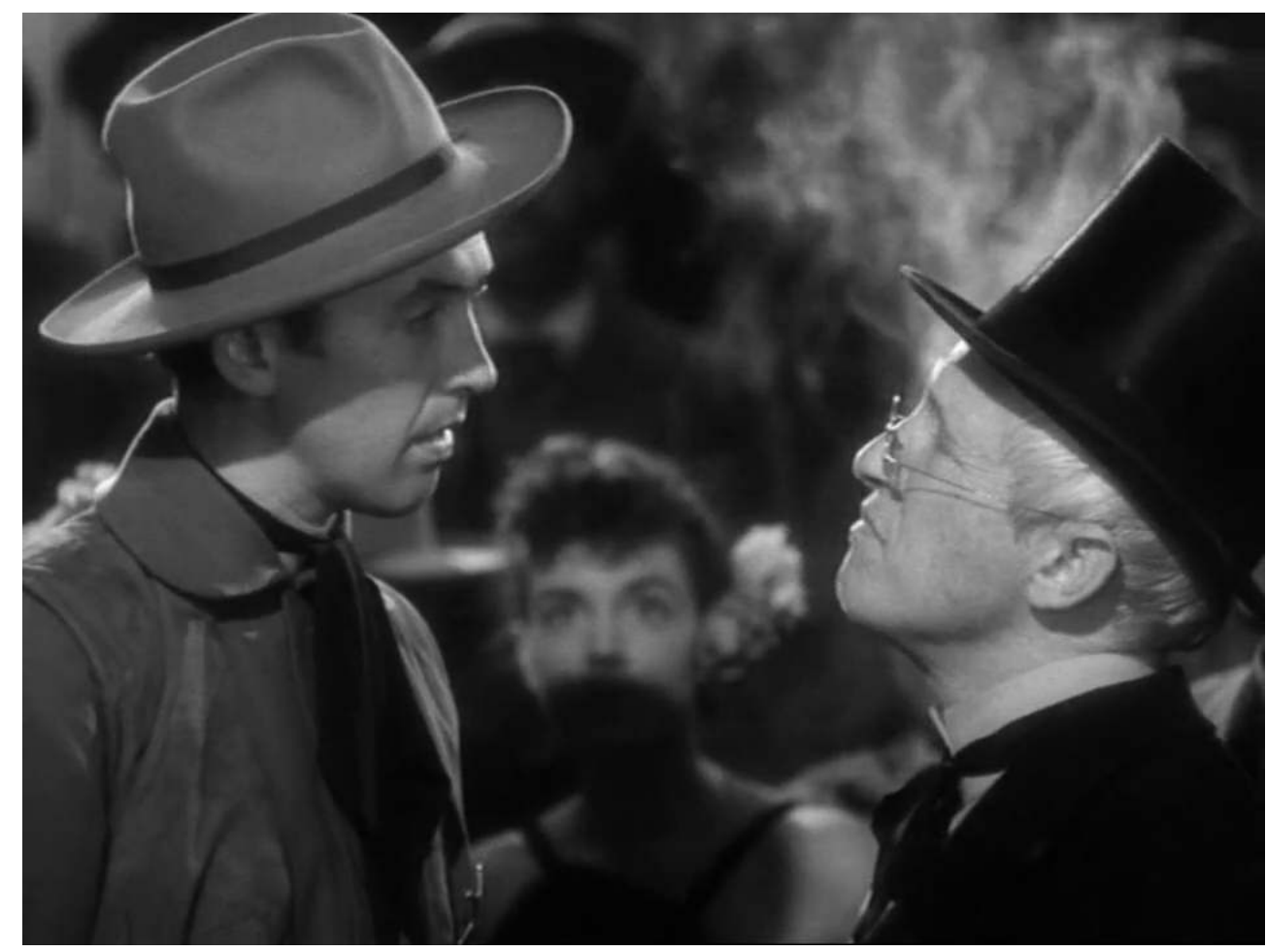

DESTRY RIDES AgAIN (GEORgE MARSHALL, 1939) : DESTRY JAUgE DU REgARD LA CORRUPTION DU MAIRE.

Le regard est tout aussi central que la voix dans Broken Arrow. Dans un film qui fait de l'homme de l'Ouest un témoin, celui dont nous allons suivre l'initiation dans le monde des Apaches, une large partie du discours pro-Indien de Broken Arrow transparaît à travers une dramaturgie du regard. Ceci est thématisé quand Milt accepte de laisser Jeffords utiliser son bureau pour les cours d'Apache et dit: "It's your eyes. " Stewart est d'abord celui qui rencontre l'autre, à travers le jeune Apache qu'il sauve qui lui apprend que son frère et sa sœur sont morts à Big Creek en 1879 (Fig. 6) ; il est ensuite le témoin de la vengeance des guerriers apaches envers ceux qui les ont scalpés ("And they made me watch the ants come. »); de retour à la ville de l'Ouest, alors même qu'il fait part de sa lassitude des guerres indiennes, il s'agace des discours racistes qu'il entend à table (Fig. 7); il découvre ensuite les traditions apaches, entreprend une relation interraciale par l'intermédiaire d'un miroir, et enfin assiste à l'embuscade tendue par un groupe de blancs qui bafouent le traité de paix. Clairement, la narration, à travers des gros plans et plans rapprochés et des compositions centrées sur les yeux, nous invite à nous aligner sur le regard du personnage. Cette puissance du regard fait de Jeffords l'égal des Apaches dont son professeur Juan dit : «Apache eyes are quick. » et surtout de Cochise : « His eyes will see into your heart. » 
Fig. 6

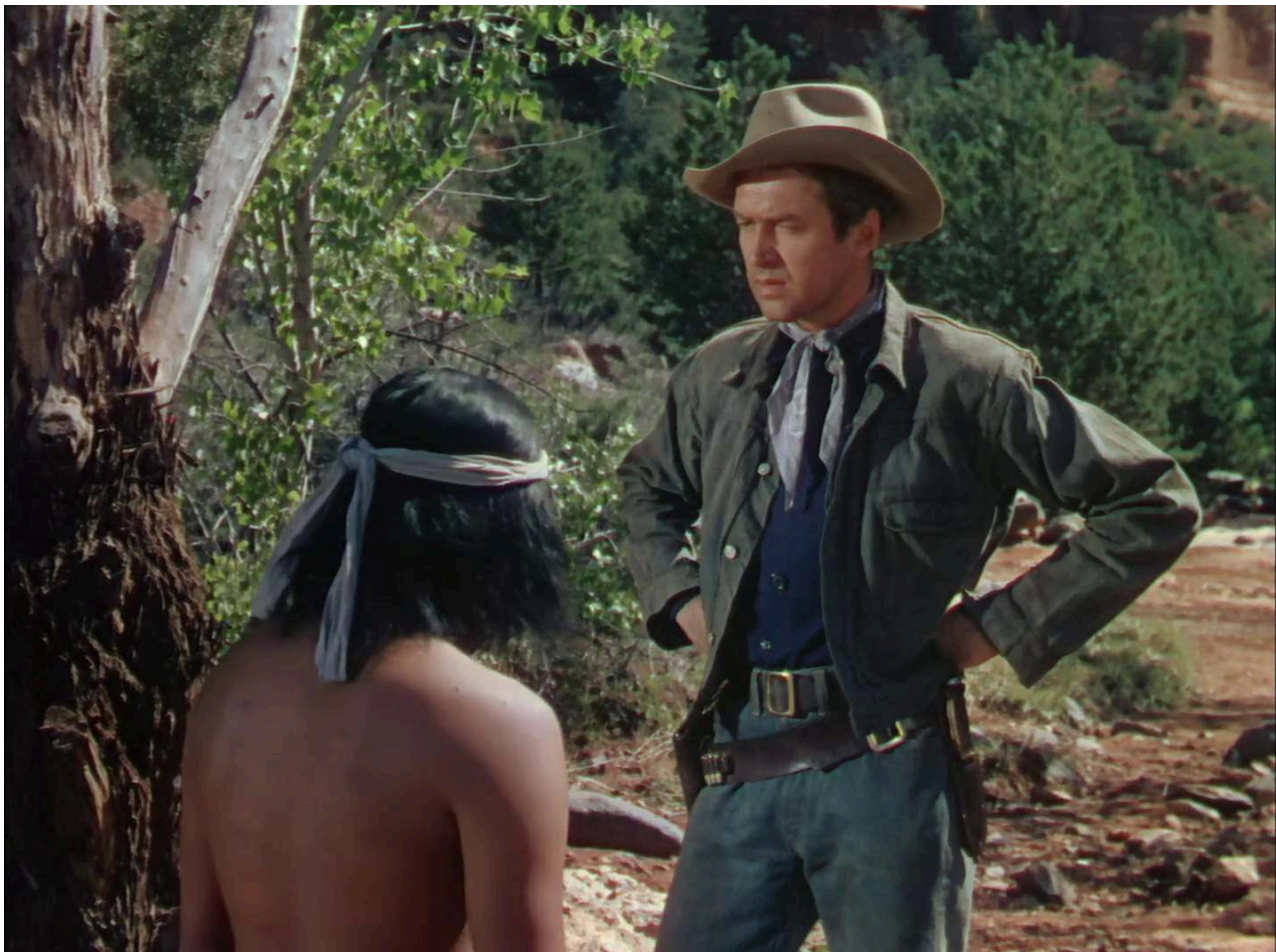

BROKEN ARROW (DELMER SAVES, 1950) : JEFFORDS PREND CONSCIENCE DE L'HUMANITÉ DU JEUNE APACHE.

Fig. 7

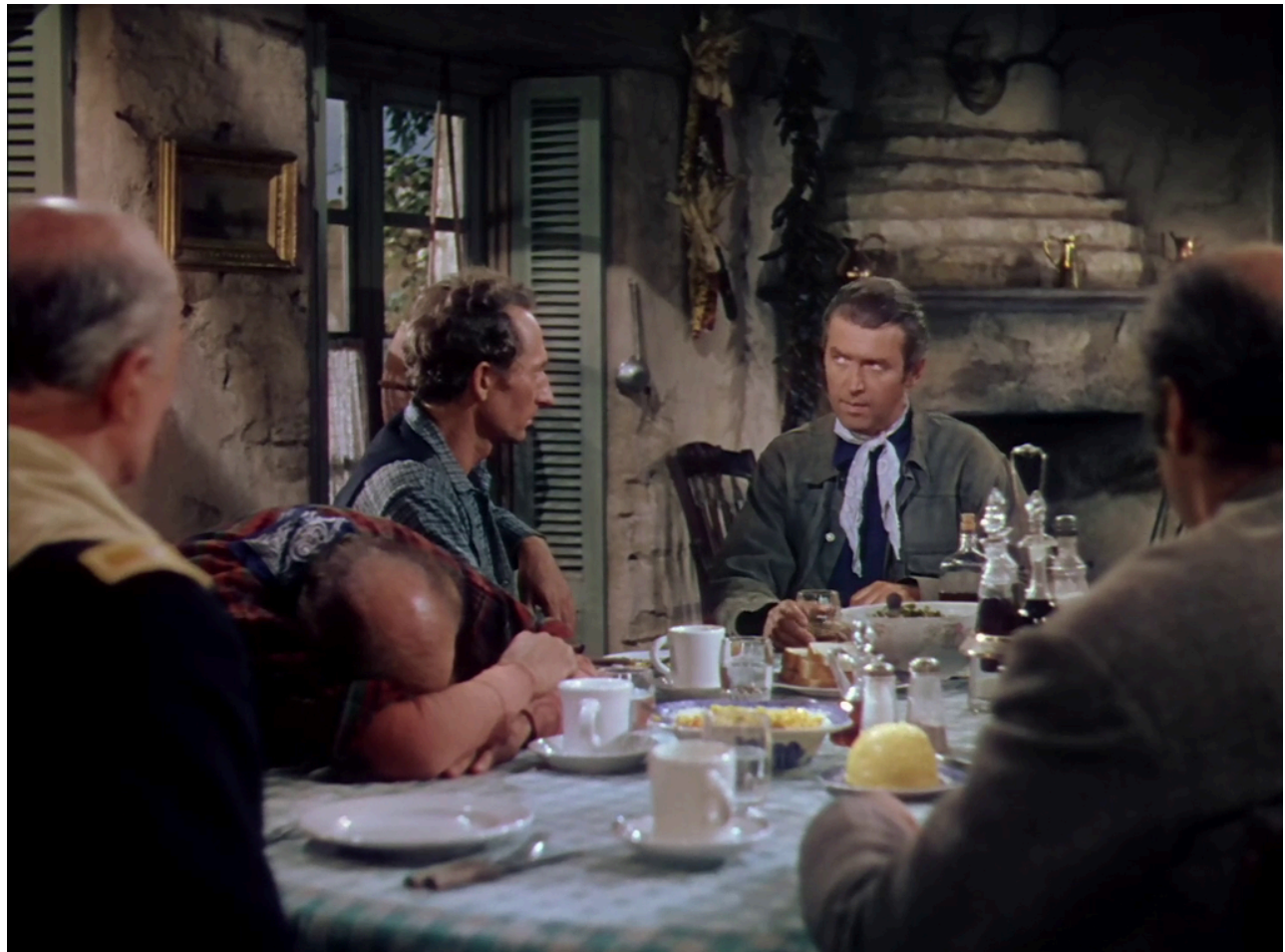

BROKEN ARROW (DELMER SAVES, 1950) : JEFFORDS MESURE LE RACISME DE SES CONCITOYENS. 
Le regard de Stewart est tout aussi central dans The Naked Spur, dans lequel il s'associe à la voix dans la critique de l'homme de l'Ouest. Stewart adopte un regard perçant et nerveux qui exprime la méfiance au gré de ses rencontres avec des inconnus (Tate, Roy). Mais ce regard s'avère rapidement lié à un manque de contrôle plutôt qu'à une maîtrise, lié au malaise du personnage de Kemp quant au statut qu'il a adopté suite à la séparation avec sa bien-aimée ; en témoignent les regards furtifs qu'il lance lorsque Ben révèle que Kemp est un chasseur de primes et non un homme de loi, et surtout le regard coléreux quand il provoque Ben en duel dans une scène qui se clôt justement sur le dos de Stewart (et donc sur la honte du personnage). Ce jeu du regard comme miroir d'une psyché traumatisée culmine par deux fois, de manière significative au contact avec Linda : au cœur du film quand il délire et la confond avec sa fiancée perdue Mary (Fig. 8), et à la fin du film quand il tente désespérément de résister à la rédemption que propose Linda en s'efforçant de maintenir coûte que coûte sa persona de chasseur de primes cupide. Dans The Naked Spur, le regard de l'homme de l'Ouest n'est pas le garant de valeurs nobles; il est le symptôme d'un trauma de guerre. Le film nous invite ainsi à nous distancer de la star masculine pour nous aligner sur la star féminine, Janet Leigh, qui de témoin devient femme salvatrice. Leigh est ainsi celle qui apaise le jeu de Stewart, celle qui nous permet de retrouver Mr. Smith et Destry.

Fig. 8

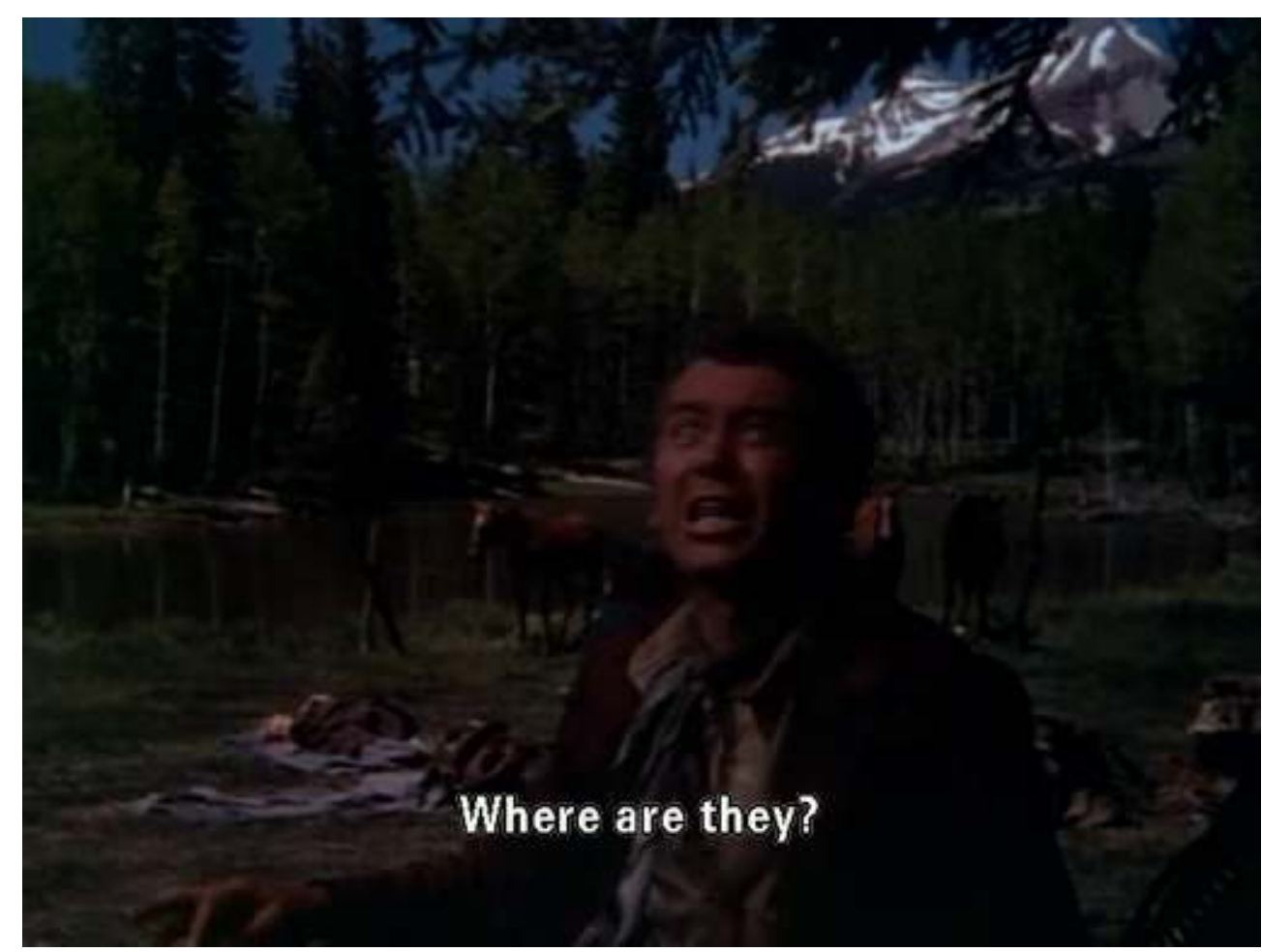

THE NAKED SPUR (ANTHONY MANN, 1953) : KEMP SE RÉVEILLE DÉLIRANT.

Les films de Ford, contrairement à ceux de Mann et d'Hitchcock, ne puisent pas tant dans le registre pathologique de Stewart, mais ont en commun avec les films de Mann que la critique est dirigée vers le personnage incarné par Stewart. The Man Who Shot Liberty Valance contraste le Sénateur aguerri qui, comme Lincoln et comme le jeune Destry, baisse les yeux quand il raconte ses histoires, avec le jeune Stoddard, au regard déterminé et courageux, qui n'hésite pas à affronter Liberty Valance dès leur première 
rencontre. Mais ce regard à la fois révolté par les mœurs de l'Ouest et généreux envers ses habitants est, in fine, un regard aveugle : il est le regard de l'homme de l'Est qui ne voit pas les limites de son discours, qui s'est trompé de lieu et de public, et qui n'est pas l'homme qui a mis fin au hors-la-loi, mais celui qui a bénéficié du regard bienveillant de l'homme de l'Ouest.

Cheyenne Autumn prolonge cette idée de l'aveuglement du personnage de Stewart, mais sur un registre plus comique. La scène de Dodge City, dont la place centrale dans la structure narrative vise à déconstruire le genre de l'intérieur, fait l'objet d'un « running joke » : Earp ne reconnaît pas Miss Plantagenet jusqu'au moment où il avoue à Doc Holliday : «By golly, I did know her in Wichita ». L'aveuglement (réel ou feint) d'Earp est présenté dès le début de la scène quand il ne réalise pas qu'il détient un as et cligne des yeux après s'être exclamé : "Oh, is that an ace? I must be blind as a bat. » (Fig. 9) Pendant le reste de la scène, Stewart, plus intéressé par le jeu de poker que par son rôle de Marshall, balaye du regard les humains (les autres joueurs, Miss Plantagenet), signalant une indifférence aux antipodes du regard plein de compassion associé à Stewart dans Mr. Smith, Destry Rides Again et Broken Arrow. Ici, son regard suspicieux est dirigé contre les autres joueurs, et donc sur sa défaite éventuelle ; même provoqué en duel, il n'a de temps que pour un bref coup d'œil alors même qu'il lâche un : « I don't doubt your word, I just question your eyesight. »

Fig. 9

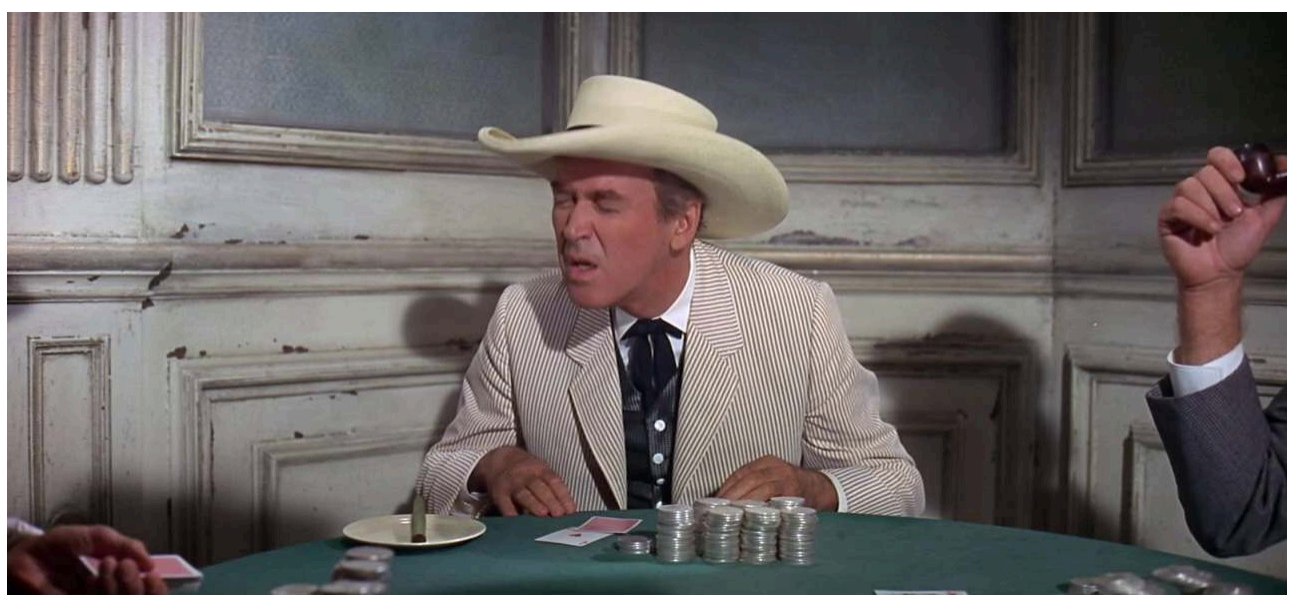

CHEYENNE AUTUMN (JOHN FORD, 1964) : WYATt EARP AVOUE ÊTRE AUSSI AVEUgLE QU'UNE CHAUVESOURIS.

\section{Conclusion}

Si l'évolution générale de la carrière de Stewart semble le mener du « folksy » vers une image plus ambiguë, voire névrotique, il serait plus juste de la considérer comme une exploration des possibles de l'acteur lui-même, aussi bien dans son jeu que dans son corps à proprement parler. Le jeu de Stewart repose indéniablement sur des caractères physiques - corps filiforme, regard perçant, grain de voix chaleureux - qui traversent sa filmographie par-delà les réalisateurs et les studios et deviennent signifiants dans un contexte donné (jeu, situation, récit). Ces éléments du jeu, que j'ai séparés pour faciliter l'analyse et pour mettre en avant les ressorts spécifiques de chacun, constituent en effet un ensemble; comme les différents instruments d'un orchestre (Moullet décrit 
fort justement Stewart comme un «homme-orchestre» [131]), comme les lignes harmoniques et rythmiques, elles peuvent s'allier, s'opposer, se nuancer. La constante, il me semble, réside dans la droiture morale - la dimension lincolnienne - associée à l'image de Stewart, et que posture, regard et voix contribuent à construire. Mais ces mêmes éléments peuvent tout aussi bien exprimer les failles dans cette droiture. C'est ainsi que, si Stewart incarne dans un premier temps Mr. Smith dans l'Ouest, celui qui accuse, celui qui repère l'injustice sur la Frontière et qui s'élève contre elle avec bon sens, courage et émotion, il devient chez Mann et chez Ford l'objet même du questionnement, l'icône - qu'il soit homme de l'Ouest ou de l'Est - que le film déconstruit, questionnement que l'échec de Destry à éviter la violence annonçait dans une moindre mesure. Plus précisément, la star vieillissante est capable d'incarner de multiples aspects parfois contradictoires de concert, non seulement du fait de son immense talent d'acteur, mais aussi du fait que son image a une histoire et que son corps en est tout imprégné. L'analyse de l'acteur est ainsi un outil précieux pour qui veut cerner la politique d'un film. Et force est de constater que, dans le cas d'une star, jeu et image entretiennent une relation dialogique et dynamique : le jeu d'une star ne relève pas uniquement des techniques actorales employées ; c'est aussi un jeu avec son image qui dépasse les éventuelles références intertextuelles.

\section{BIBLIOGRAPHIE}

Agnel, Aimé. L'Homme au tablier. Le jeu des contraires dans les films de Ford. Rennes : La Part Commune, 2002.

Chabrol, Marguerite. De Broadway à Hollywood : stratégies d'importation du théâtre new-yorkais dans le cinéma classique américain. Paris : CNRS éditions, 2016.

Coe, Jonathan. James Stewart. Une biographie de l'Amérique. Traduit par Béatrice Pley. Paris : Cahiers du cinéma, 2004 [1994].

Damour, Christophe. «L'Influence de Delsarte sur le jeu de l'acteur de cinéma aux États-Unis ». In L'Acteur de cinéma : approches plurielles. Eds. Vincent Amiel, Jacqueline Nacache, Geneviève Sellier et Christian Viviani. Rennes : PU Rennes, 2007, p. 19-31.

Davis, Alex. « The Star Auteur: Jimmy Stewart Out West ». In Unbridling the Western Film Auteur: Contemporary, Transnational and Intertextual Explorations. Eds. Emma Hamilton et Alistair Rolls. Oxford: Peter Lang, 2018, p. 25-44.

Dyer, Richard. Stars. Londres : BFI, 1998 [1979].

Eliot, Marc. Jimmy Stewart: A Biography. New York: Three Rivers Press, 2006.

Mayer, Hervé. La construction de l'Ouest américain dans le cinéma hollywoodien. Neuilly : Atlande, 2017.

Moullet, Luc. Politique des acteurs : Gary Cooper, John Wayne, Cary Grant, James Stewart. Paris : Éditions de l'Étoile / Cahiers du Cinéma, 1993. 
Naremore, James. Acteurs : le jeu de l'acteur au cinéma. Traduit de l'anglais par Christian Viviani. Rennes : PU Rennes, 2014 [1988].

Peek, Wendy Chapman. «The Romance of Competence: Rethinking Masculinity in the Western». Journal of Popular Film and Television 30.4 (2003), p. 206-19.

Pomerance, Murray. « James Stewart and James Dean: The Darkness Within». In Larger than Life: Movie Stars of the 1950s. Ed. R.B. Palmer. New Brunswick: Rutgers University Press, 2010, p. 61-85.

Sklar, Robert. Movie-Made America: A Cultural History of American Movies (Revised and Updated). New York : Vintage Books, 1975, 1994.

Slotkin, Richard. Gunfighter Nation: The Myth of the Frontier in Twentieth-Century America. Norman: U of Oklahoma P, 1998 [1992].

Viviani, Christian. Le magique et le vrai : L'acteur de cinéma, sujet et objet. Aix-en-Provence : Rouge Profond, 2015.

\section{NOTES}

1. C'est en ces termes que Luc Moullet décrit la carrière de Stewart : « On a ainsi un parcours très surprenant: la pureté naïve du jeune Smith, créé par Capra, qui luttait pour le bien public, ensuite le chacun pour soi, l'absence de toute solidarité manifestée à travers la violence (chez Mann) ou la passivité de l'adulte (chez Hitchcock), enfin vers les cinquante ans, le cynisme et la vénalité (chez Ford), on a là une progression négative qui contraste en tous points avec la carrière des trois autres grands. » (137)

2. Texte original : «What emerges in Stewart's character is not the genteel Destry, but rather the determined ferociousness of Mr. Smith on the Senate floor, this time channeled into a need for vengeance ".

3. L'historien Richard Slotkin explique que, dans les romans de James Fenimore Cooper par exemple, les attitudes sur la question des guerres indiennes étaient conçues en termes genrés : "the opposition between "hard" and "soft" understandings of social and class questions embodied in the gendered contrast of Masculine and Feminine ways of thinking about Indian wars » (15-16).

\section{RÉSUMÉS}

Cet article propose de déterminer dans quelle mesure le jeu et la persona de James Stewart participent au discours politique de cinq Westerns, Femme ou démon (Marshall, 1939), La Flèche brisée (Daves, 1950), L'Appât (Mann, 1953), L'Homme qui tua Liberty Valance (Ford, 1962) et Les Cheyennes (Ford, 1964). L'analyse de la posture, de la voix et du regard révèle que la critique est de plus en plus dirigée envers les figures incarnées par Stewart (qu'il s'agisse de l'homme de l'Ouest ou de l'Est) plutôt que contre les forces auxquelles il s'oppose. Cette étude suggère que, dans le cas d'une star, jeu et image entretiennent une relation dialogique et dynamique : le jeu d'une star ne relève pas uniquement des techniques actorales employées ; c'est aussi un jeu avec une image. 
This article proposes to assess to what extent James Stewart's acting and star image participate in the politics of five Westerns, Destry Rides Again (Marshall, 1939), Broken Arrow (Daves, 1950), The Naked Spur (Mann, 1953), The Man Who Shot Liberty Valance (Ford, 1962) and Cheyenne Autumn (Ford, 1964). Attention to posture, voice and looks reveals that the critique is increasingly directed at the figures Stewart embodies, be it the Man of the West or the East, rather than those he confronts. The study suggests that, in the case of a star, acting is as much an engagement with a specific role as with the star's image.

\section{INDEX}

Keywords : James Stewart, Western, acting, star image, politics, ideology, masculinity, Delmer Daves, John Ford, Anthony Mann, George Marshall, Destry Rides Again, Broken Arrow, The Naked Spur, The Man Who Shot Liberty Valance, Cheyenne Autumn

Mots-clés : James Stewart, Western, jeu d'acteurs, star, politique, idéologie, masculinité, Delmer Daves, John Ford, Anthony Mann, George Marshall, Femme ou démon, La Flèche brisée, L'appât, L'homme qui tua Liberty Valance, Les Cheyennes

\section{AUTEURS}

\section{DAVID ROCHE}

Université Toulouse Jean Jaurès

mudrockca@gmail.com 